\title{
Retention Rate of Customers in Banks using Neural Networks
}

\author{
T. H. Feiroz Khan, Shrushti Mhaske, Sonal Yeshwantrao, Ayush Kumar
}

\begin{abstract}
Customer retention is the process of retaining the customers after a particular period of time. Profitability of a product depends upon the retention of the customer. In the earlier systems a lot of money, time and resources were spent on advertising by banks. Banks approached call centres to try convince the customers in buying new policies. This didn't really work since most of it was directed towards untargeted customers. This same problem of customer retention is studied and a competent solution is found. This system consists of analysed data of a number of customers in numerous fields and checks the retaining rate of every customer. Use of a neural network is advocated to check past conditions of services provided to a customer by the banks and a common retainability index is produced which predicts the retention rate of the new customers.
\end{abstract}

Keywords: Neural Networks, Prediction, Machine learning, Retention Rate.

\section{INTRODUCTION}

Now a days, banks face a lot of competition in providing facilities to their customers. Banks get the details of a loyal customer and then provide new offers to the customer in order to retain them. Large banks use the past data to predict the similarities and the use in to retrieve customer. In India, commercial banks have asset of 40,900,00 million rupees and a GDP 65\% as of 2019. The countries structural foundation growth is made by the banking corporations. Banks need to be updated with the latest technologies. State Bank of India lost 4.16 million customers in the year 2016-2017 thus facing a huge loss. These problems arise due the inability of banks to satisfy the customer needs and expectations. Most of the banks are in profits due to their loyal customers and if they lose their customers it would result in futile outcomes.

Revised Manuscript Received on October 30, 2019

* Correspondence Author

Mr.T.H. Feiroz Khan*, Computer Science and Engineering, SRM Institute of Science and Technology, Chennai, India. Email: feirozkh@srmist.edu.in

Shrushti Mhaske, Computer Science and Engineering, SRM Institute of Science and Technology, Chennai, India. Email: Shrushti1704@gmail.com

Sonal Yeshwantrao, Computer Science and Engineering, SRM Institute of Science and Technology, Chennai, India. Email: sonalyeshwantrao56@gmail.com

Ayush Kumar, Computer Science and Engineering, SRM Institute of Science and Technology, Chennai, India. Email: ajayush786@gmail.com

(C) The Authors. Published by Blue Eyes Intelligence Engineering and Sciences Publication (BEIESP). This is an open access article under the CC BY-NC-ND license (http://creativecommons.org/licenses/by-nc-nd/4.0/) Accuracy, Data analysis, Linear regression, Dataset, Training,

\section{NEURAL NETWORKS}

Neural networks are biological neurons. They are sort of computer software. The cells in Neural networks are called nodes and they work together to produce the required result. Each node is responsible to solve only a small part of the problem. It is an example of machine learning.

\section{LINEAR REGRESSSION}

Linear Regression is a method of finding a line that is the best and fits the points consisting of data on plots. In this way we can predict the output values even if the inputs are not present in our dataset. This is done by assuming that those outputs will fall on the line. In this the points refer to the neural nodes

\section{EXISTING SYSTEM}

- Bank hire call centres to try to convince customers to buy new policies.

- Lot of money, time and resources are spent by banks on advertising which is mostly directed towards an untargeted customers.

- Due to scarcity of resources in rural areas, the majority of population remains untargeted and unserved.

- Data pre-processing is not done.

- There arises a need of individual analysis of a customer but proper solution is found.

\section{OBJECTIVE OF THE SYSTEM}

The previous data sets are studied by neural network lines and the outcome is predicted. Machine learning uses neural networks and provides outcome by analyzing large data and thus helps the bank to be financially successful. The predictions obtained by this system will allow the banks to provide new policies and schemes to the customers probably leaving the bank and thus retain the customer. The final output of the retention rate depends upon the nature of the neural networks. The input layer which consists of neural networks has n number of terminals. The second hidden layer consists of multiply input layers with respect to their weightage in the model. The final output depends on the nature of the networks. The final output layer has a result which depends upon the nature of the neural networks. The

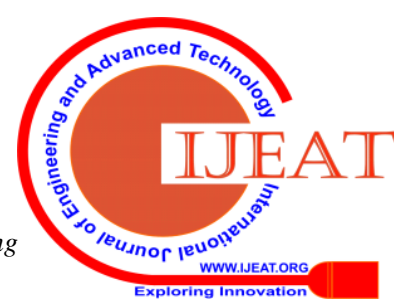


output layer has a predictive analysis of the input sets. Artificial neural networks (ANN's) consist of functions such as training and testing. ANN uses the previous data set to train itself and then the trained ANN is tested on a new set of data. The ANN monitors throughout the process to check for any errors. Back propagation method is used to correct any errors if found through the neural network.

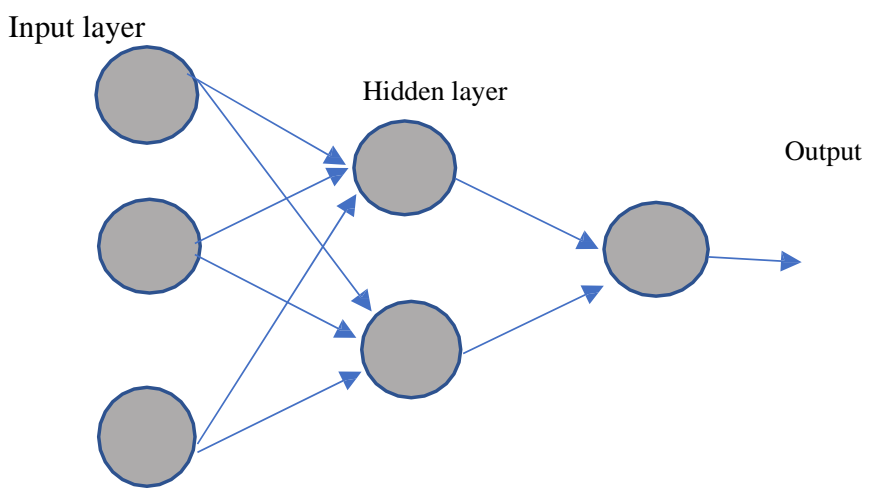

Fig 5.1 Functionality of a Neural Network

\section{ADVANTAGES OF THE SYSTEM}

The biggest advantage in this proposed system is the use of neural network. The use of neural network to solve this complex problem makes the model efficient to produce high percentage of accuracy. Neural network is not totally dependent upon the input data to predict the output, it has self-learning ability. Therefore, neural network is widely used in real time applications. The network gets trained by the sample dataset and then it can be applied to similar affairs. It is capable of learning and deriving relationships between complex and non-linear data. Detection of errors in the system is not difficult and multiple tasks can be performed in parallel without affecting the system efficiency. Data can be regenerated from data sets in neural networks. Our motive is to apply this proposed system in strengthening customer relationship management.

\section{SYSTEM ARCHITECHTURE}

This model is capable of working with different facets and branches. The different branches are the criteria which have a weightage to give an output accordingly. There exists two components $\mathrm{X}$-axis and $\mathrm{Y}$-axis. The branches are marshalled as a component of $\mathrm{X}$-axis and the exiting condition of a given customer is marshalled as y-axis. Only the criteria which affect the result are chosen for the branches.

The data is divided into training data and the test set to overcome over-training problems and making the system too specific to the sample data.

In this, $80 \%$ of the data is used for the training purpose and the rest $20 \%$ is used for the test set.

The training data is continuously passed through the neural network and each time it passes forward and backward, the network becomes more accurate and efficient. After this the system is checked for its accuracy. The test set is used for evaluation, it checks the performance of the current neural network and shows if the model is acceptable.
If the input has $\mathrm{x}$ neurons and output has $\mathrm{y}$ neurons then the hidden layer has $(x+y) / 2$.

\section{REAL TIME APPLICATION}

A dataset of 10,000 random customers is used to show the implementation of this proposed system. There are total 13 labels under which the dataset is grouped which are called the branches. Out of these 13 only 2 branches do not hold any weightage in the output. The branches include age, activity, balance, credit score, tenure, number of total products used, income, gender, geography and the condition whether the customer has exited or not. The data is passed 25 times through the neural network and the predicted accuracy is $83.61 \%$. The predicted rate is decent and therefore useful in real time. Accuracy of training set: $86.56 \%$

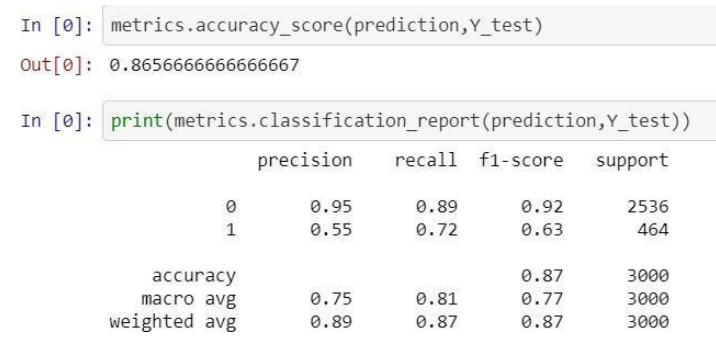

Fig 8.1 Precision rate

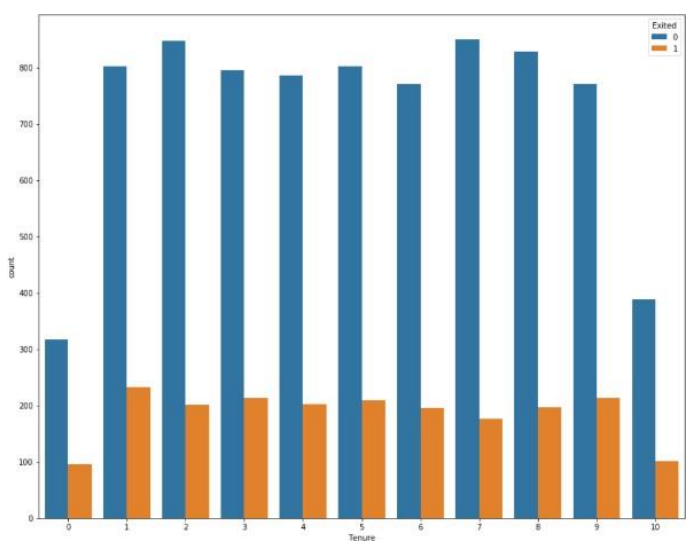

Fig 8.2 Bar graph plot

The above bar graph is a graph consisting of tenure of the customers in the bank. The bars are the exited values of the customers. The orange bar i.e. 1 is of the customers who have left the bank and the blue bar i.e.0 is of the customers that have been retained. 


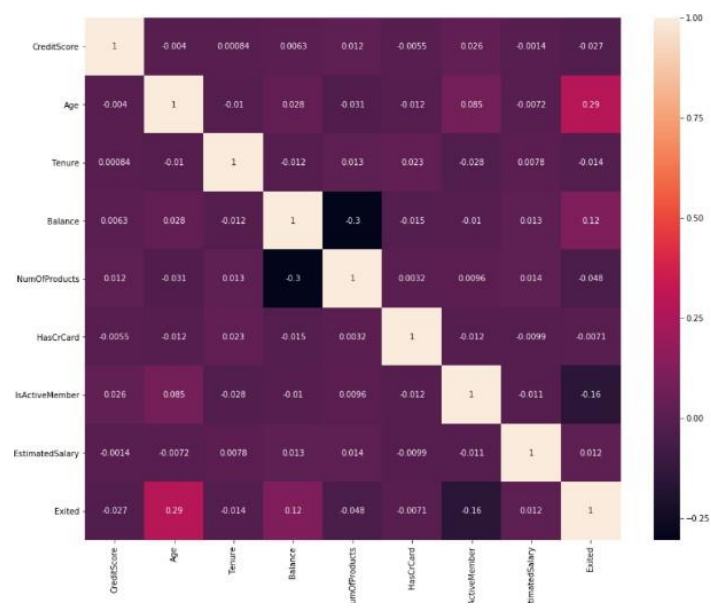

Fig 8.3 Correlational graph plot

The above graph is a correlational graph which consists of the various factors from the data set such as credit score, age, tenure, balance, etc. It determines the relation between entities in order to predict the accuracy over test set.

\section{CONCLUSION}

This proposed system is designed to solve a complex problem that is predicting the retention rate of customers in banks. This system provides a high level of accuracy and the retainability rate can be predicted efficiently. The use of neural networks makes this model easy to use and implement in the real world. Therefore a our motive of solving the major problem faced by banks these days is successfully solved.

\section{REFERENCES}

1. Alisa Bilal Zoric, "Predicting Customer churn in Banking Industry using Neural Networks",

2. Adnan Amin, Sajid Anwar, Awais Adnan, Muhammad Nawaz, Newton Howard, Junaid Qadir , Ahmad Hawalah, Amir Hussain,"Comparing oversampling techniques to handle the class imbalance problem: A customer churn prediction case study"

3. Andreas Krause and Simone Giansante, "Network - Based Computation Techniques to Determine the Risk Drivers of Bank Failures During a Systemic Banking Crisis.

4. Chih-Fong Tsai and Yu-Hsin Lu, "Data Mining Techniques in Customer Churn Prediction".

5. Dudyala Anil Kumar and V.Ravi ,"Predicting Credit card customer churn in banks using Data mining ",Data analysis techniques and Strategies.

6. Fang Wang ,Hua Li, Aijun Liu and Xiao Zhang, "Hybrid customer requirements rating method for customer - oriented product design using QFD".

7. Jungsuk kwac ,Jung In Kim and Ram Rajgopal , "Efficient customer selection process for various DR objectives ".

8. Dr. Prabin Kumar Panigrahi and Anuj sharma ,"A Neural Network approach for predicting Custoomer Churn in Cellular Network Sevices ".

9. Xishun Wang, Minjie Zhang and Fenghui Ren , "Learning Customer Behaviour for Effective Load Forecasting".

10. Zolidah Kasiran, Zaidah Ibrahim and Muhammad Syahir Mohd Ribuan, "Customer Churn Prediction using Recurrent Neural Network with Reinforcement Learning Algorithm in Mobile phone users".

\section{AUTHORS PROFILE}

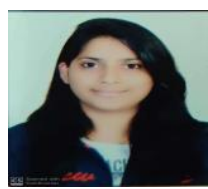

Shrushti Rohitdas Mhaske is a 3rd year student, currently pursuing her B.Tech degree in Computer Science and Engineering from the SRM Institute of Science and Technology, Ramapuram, Tamilnadu. She did her schooling from St.Felix High School, Pune and did her Higher Secondary Course in the field of Science with specialisation in Electronics from Nowrosjee Wadia College, Pune. She aspires to become a Machine Learning Engineer and her dream company is Google. Her research interests include Machine Learning, Neural Networks and Artificial Intelligence

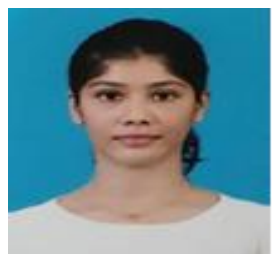

Sonal Rajesh Yeshwantrao is a 3rd year student, currently pursuing her B.Tech degree in Computer Science and Engineering from the SRM Institute of Science and Technology, Ramapuram, Tamilnadu. She did her schooling from St.Helena's High School, Pune and did her Higher Secondary Course in the field of Science with specialisation in Computer Science from Sardar Dastur Hormazdiar Junior College, Pune. She is currently preparing for GRE and plans to do her masters in Data Analytics from the United States of America. Her interests include handling SQL databases and other research interests include Neural Networks and Machine Learning.

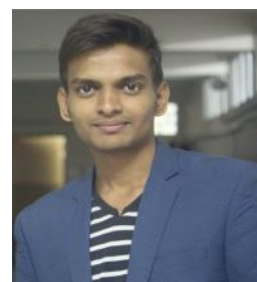

Ayush Kumar is a 3rd year student, currently pursuing his B.Tech degree in Computer Science and Engineering from the SRM Institute of Science and Technology, Ramapuram, Tamilnadu. $\mathrm{He}$ did his schooling and Higher Secondary Course from Patna, Bihar. He aspires to become a Data Engineer and plans to start his own startup company in the year 2021. Apart from this, he has successfully completed various courses in Machine Learning and Artificial Intelligence. His other research interests include Neural Networks and Robotics.

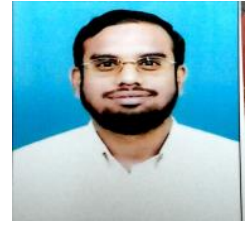

Mr. T. H. Feiroz Khan received his M.E. degree in Computer science Engineering from Annamala University,Tamilnadu in 2004. Currently pursuing $\mathrm{PhD}$ in school of computing in the Domain of Wireless Networking. He is a currently Assistant Professor in SRM Institute of Science and Technology, Chennai. He has teaching work experience of 15 years in the Engineering and Technology. He is member of International Association of Engineers (IAENG).His research interest includes Wireless System and Machine Learning. 